\title{
Cooperative transport in nanochannels
}

\author{
Wolfgang R. Bauer ${ }^{1, *}$ and Walter Nadler ${ }^{2, \dagger}$ \\ ${ }^{1}$ Department of Internal Medicine I, University Hospital of Würzburg, Oberdürrbacher Straße 6, D-97080 Würzburg, Germany \\ ${ }^{2}$ Institute for Advanced Simulation, Jülich Supercomputing Centre, Forschungszentrum Jülich, D-52725 Jülich, Germany
}

(Received 9 November 2012; published 8 July 2013)

\begin{abstract}
Channel transport of different species of particles is viewed usually only in terms of competition and selectivity. In this paper we show that transport of one species may be promoted by the presence of another and that both may even mutually cooperate. We investigate a discretized Markovian model of nanochannel transport via in-channel sites, allowing for the simultaneous transport of several different species of particles; interaction between transported particles is included via the condition of single occupancy on a channel site. By numerically solving the model exactly, particularly an analysis of situations of crowding in the channel is possible and we observe three situations: mutual cooperation, promotion of one species at the cost of the other, and mutual competition. The physical situation has a strong nonequilibrium character as Onsager's relations on coupled flows do not hold.
\end{abstract}

DOI: 10.1103/PhysRevE.88.010703

PACS number(s): 05.60.-k, 87.16.dp, 66.10.cg, 87.10.Mn

Particle transport through channels is of paramount importance in many fields, ranging from life sciences to nanotechnology $[1,2]$. Two main questions were addressed in the past: How is optimal transport achieved and how can a channel provide selectivity? Optimal transport was shown to depend on a tuning of particle-channel interactions, e.g., binding or entropic trapping [3-7], channel blocking and crowding as a result of interparticle interaction [8-11], and particle-channelend interaction $[12,13]$. Selectivity means that a channel favors transport of one species, which competes for channel access with other species. This can be achieved by differential channel affinities, as simulations [14] and analytical models [12] have demonstrated. However, selectivity implies that one species is favored by a stronger in-channel affinity at the cost of the other, i.e., one has an asymmetric competitive scenario. In this paper we will address whether there may also be promotion of one species by the other and whether there may be even cooperation of species of particles. Analysis is performed in a spatially discrete model of channel transport of two species that is exactly solvable numerically. It explicitly conserves the spatial correlations related to interparticle interaction and does not average them out, as it is done in mean field approaches.

We consider the transport of two species $A$ and $B$ through a channel that connects two baths 1 and 2 with respective particle concentrations of each species inside, $\left(c_{1, A}, c_{2, A}\right)$ and $\left(c_{1, B}, c_{c, B}\right)$. The driving forces inducing transport through the channel are the concentration gradients. In addition, transport is affected by interparticle and particle-channel interactions. A discretized model of the spatial channel positions, labeled by $i=1, \ldots, N$, is used. Interparticle interaction is reflected by the fact that one spatial position may only be occupied by one particle, i.e., a particle inside the channel can only move to a vacant spatially neighboring site. The respective particle-channel interactions are described by a potential $\Phi_{i}^{(A, B)}$. Outside the channels, i.e., in the baths, the potential is set to zero. The transition rate for a particle of species $(A, B)$

\footnotetext{
*Corresponding author: w.bauer@medizin.uni-wuerzburg.de ${ }^{\dagger}$ w.nadler@fz-juelich.de
}

to jump from position $i$ to a free neighboring position $i \pm 1$ is then given by [15]

$$
r_{i \rightarrow i \pm 1}^{(A, B)}=1 / \tau_{0}^{(A, B)} \exp \left[-\left(\Phi_{i \pm 1}^{(A, B)}-\Phi_{i}^{(A, B)}\right) / 2\right],
$$

where the time constant $\tau_{0}$ determines the mobility of the particle, which is assumed to be constant within the channel. Under equilibrium conditions detailed balance holds, $r_{i \rightarrow i \pm 1} / r_{i \pm 1 \rightarrow i}=e^{-\left(\Phi_{i \pm 1}-\Phi_{i}\right)}$. Note that we normalize energetic quantities to $k T$, e.g., $\Phi / k T \rightarrow \Phi$, with the Boltzmann constant $k$, and time to $\tau_{0}$, i.e., $t / \tau_{0} \rightarrow t$, which leaves energetic parameters as well as flows and rates dimensionless.

We assume symmetric exchange dynamics at the channel ends. Hence the rates with which particles enter a vacant entrance position of the channel from the bath 1 or 2 with concentration $c_{1}^{(A, B)}$ or $c_{2}^{(A, B)}$ can be defined as

$$
\begin{aligned}
r_{\text {bath } 1 \rightarrow i=1} & =k_{+}^{(A, B)} c_{1}^{(A, B)} e^{-\Phi_{1}^{(A, B)} / 2}, \\
r_{\text {bath2 } \rightarrow i=N} & =k_{+}^{(A, B)} c_{2}^{(A, B)} e^{-\Phi_{N}^{(A, B)} / 2} .
\end{aligned}
$$

Correspondingly, the rates with which particles leave the channel ends are

$$
\begin{gathered}
r_{i=1 \rightarrow \text { bath } 1}=k_{-}^{(A, B)} e^{\Phi_{1}^{(A, B)} / 2}, \\
r_{i=N \rightarrow \text { bath2 }}=k_{-}^{(A, B)} e^{\Phi_{N}^{(A, B)} / 2} .
\end{gathered}
$$

Note that these rates respect the vanishing potentials in the baths.

A channel state $\sigma$ is completely described by a state variable $\boldsymbol{\sigma}=\left(\sigma_{1}, \ldots, \sigma_{i}, \ldots, \sigma_{N}\right)$ where the value of its components $\sigma_{i}$ indicates whether the spatial state $i$ is either empty or occupied by one particle of species $A$ or $B$ [16]. So our state space is $\boldsymbol{\Sigma}=\left\{\boldsymbol{\sigma} \mid \boldsymbol{\sigma}=\left(\sigma_{1}, \ldots, \sigma_{N}\right), \sigma_{i}=0, A, B\right\}$ and it has dimension $3^{N}$. We consider the dynamics of this state space as a continuous Markov process. The evolution of the probabilities $\boldsymbol{P}=\left[P_{\sigma}(t)\right]_{\sigma \in \boldsymbol{\Sigma}}$ to find the channel in the respective states is then determined by a master equation

$$
\frac{d}{d t} \boldsymbol{P}(t)=\boldsymbol{\Lambda} \boldsymbol{P}(t),
$$

where the $3^{N} \times 3^{N}$ matrix $\boldsymbol{\Lambda}$ consists of the transition rates $\lambda_{\sigma \rightarrow \sigma^{\prime}}$ between channel states $\sigma$ and $\sigma^{\prime}$. These are given by the 
rates in Eqs. (1)-(3) if $\sigma^{\prime}$ can evolve from $\sigma$ by the transition of a particle from its original position to a neighboring position, under the constraint that the above-mentioned interparticle interactions hold. Otherwise the transition rate between states is zero. Particle flow between position $i$ and $i+1$ is

$$
\begin{aligned}
J_{i, i+1}^{(A, B)}(t)= & \sum_{\sigma \in \Sigma} P_{\sigma}(t)\left[r_{i \rightarrow i+1}^{(A, B)}\left|\sigma_{i}\right|\left(1-\left|\sigma_{i+1}\right|\right)\right. \\
& \left.-r_{i+1 \rightarrow i}^{(A, B)}\left|\sigma_{i+1}\right|\left(1-\left|\sigma_{i}\right|\right)\right],
\end{aligned}
$$

with $\left|\sigma_{n}\right|=1$ when the position $n$ is occupied by either species and otherwise $\left|\sigma_{n}\right|=0$. Hence the terms $\left|\sigma_{i}\right|\left(1-\left|\sigma_{i+1}\right|\right)$ ensure that a given state $\sigma$ contributes only to flow when the position $i$ is occupied and $i+1$ is empty and vice versa in the reverse direction. The above equation also holds for flow at the channel ends, with the modification that transitions to the baths are not restricted by the particles therein.

We consider the steady state, i.e., the probability distribution obeys

$$
\boldsymbol{\Lambda} \boldsymbol{P}_{s}=0 .
$$

Particle flow along the channel is then constant in space, i.e., $J_{i, i+1}^{(A, B)} \equiv J^{(A, B)}$ is independent of $i$. Here $\boldsymbol{P}_{s}$ and hence flow via Eq. (5) can be numerically determined exactly.

We consider two species (Fig. 1) and start from equilibrium, i.e., concentration gradients and hence flows of both vanish. Increasing the gradient of one species monotonically enhances its flow, whereas the dependence on channel length exhibits the opposite effect. For higher gradients flow of this species shows a saturation behavior, which results from channel blocking impeding flow, and is well known from single-species transport [10]. In contrast, while the concentration gradient of the second
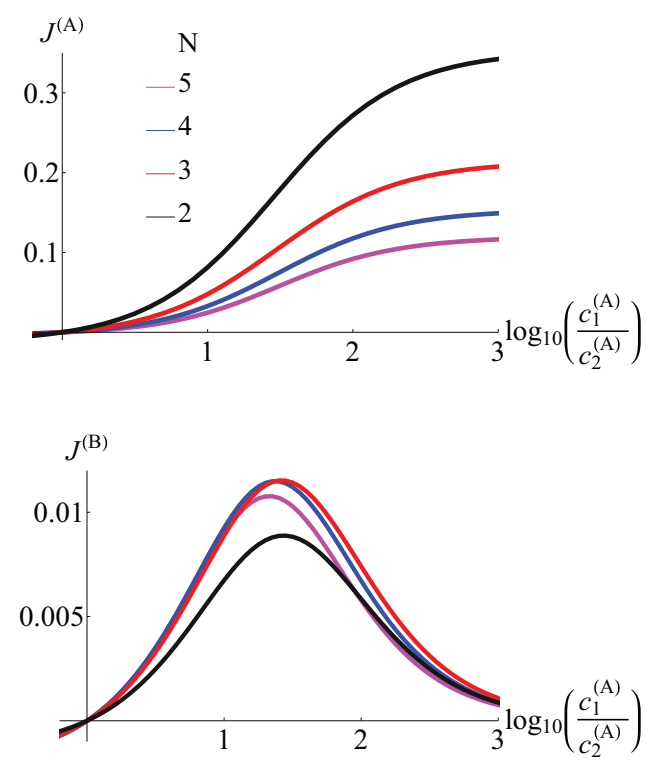

FIG. 1. (Color) Flow of the driving species $J^{(A)}$ (top) and driven one $J^{(B)}$ (bottom) vs concentration gradient of the driving species $A$, for different channel lengths $N=2, \ldots, 5$. Species $A$ is inert, i.e., $\Phi_{i}^{(A)} \equiv 0$, and its concentration in bath 2 is fixed to $k_{+} c_{2}^{(A)}=0.1$. The gradient of species $B$ vanishes, $k_{+} c_{1}^{(B)}=k_{+} c_{2}^{(B)}=0.1$, and it exhibits a constant attractive particle-channel interaction, $\Phi_{i}^{(B)} \equiv-2$. We set $k_{-}=1$ for $A$ and $B$.

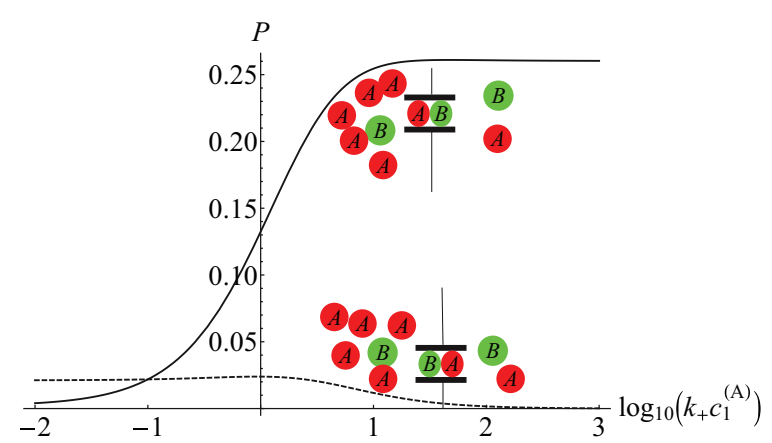

FIG. 2. (Color) Steady state probability of $\sigma=(A, B)$ (solid line) and $\varsigma=(B, A)$ (dashed line) vs the concentration of the driving species $A$ in bath 1 , for a channel length $N=2$. Other parameters are the same as in Fig. 1.

species remains zero, its flow nevertheless also increases and eventually runs through a maximum before asymptotically approaching. The latter is obviously a consequence of the eventual expulsion of the second species from the channel. Interestingly, channel length has only moderate effects on flow of the second species. In the following we will call the first species the driving species and the second the driven species.

This cooperative phenomenon, i.e., that one species induces flow of the other, has its origin in entropic forces built up by the driving species within the channel, a phenomenon also known from, e.g., osmosis $[17,18]$. The concentration gradient of the driving species favors the probability of channel states that contain both species and in which the ordering of particles of the driving and the driven species biases transitions of the latter in the direction of this gradient (see Fig. 2). In the simple case of two spatial positions within the channel, there exist only two states in which both species are present in the channel, namely, $\sigma=(A, B)$ and $\varsigma=(B, A)$.

Comparing Figs. 1 and 2, it can be seen that the increase of flow of the driven species $B$ with increasing concentration gradient of $A$ follows the rise of the probability of state $(A, B)$; in contrast, its further decrease follows the decrease of the probability of state $(B, A)$. It is the interplay of these concomitantly occupied multispecies states that leads to the flow of the driven species.

It should be emphasized that solely entropic forces induce the bias of flow of species $B$. It is also important to note that the intuitive picture that a flowing species $A$ picks up particles of species $B$ on its way through the channel, which is the base of "knock on" models of channel transport [19], is wrong, as we model the dynamics between states as a Markov process, i.e., inertia is absent, and interparticle interaction solely occurs on the base of occupied spatial positions, i.e., entropic constraints. Note that the cooperation of two species demands the interaction of both within the channel, i.e., the channel must be capable of containing at least one molecule of either species simultaneously. So, for molecules larger than the channel length this mechanism does not work.

Cooperation of two species is not limited to the situation of vanishing gradient of the driven species. Instead the driven species may even flow against its concentration gradient (Fig. 3). Zero flow of the driven species requires a sufficiently strong oppositely directed concentration gradient 


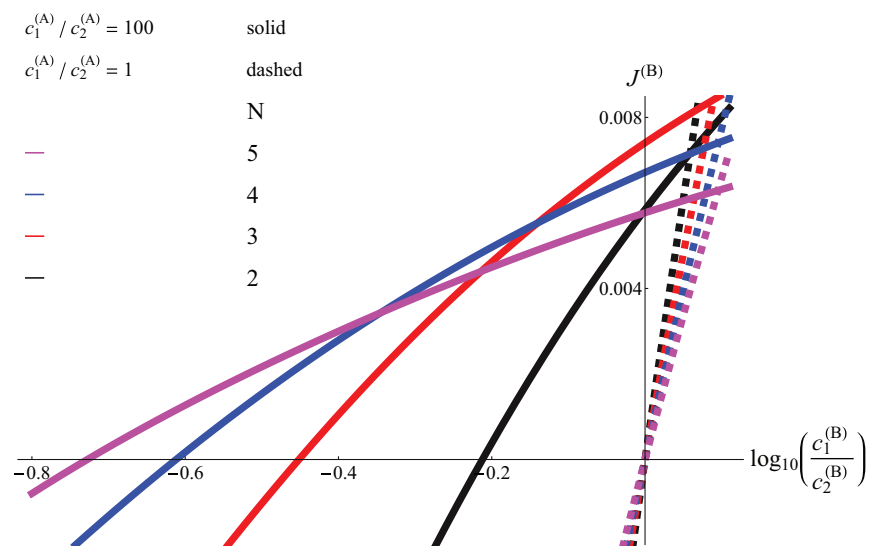

FIG. 3. (Color) Gradient of a driving species $A$ promotes flow of $B$ against its concentration gradient (solid lines) $\left(k_{+} c_{1}^{(B)}=0.1\right.$, $k_{+} c_{2}^{(B)}$ is varied, and $\left.k_{+} c_{2}^{(A)}=0.1\right)$. Other parameters are the same as in Fig. 1. In the absence of a gradient of $A$ (dashed lines), flow of $B$ simply vanishes with its concentration gradient.

$\log _{10}\left(c_{1}^{(B)} / c_{2}^{(B)}\right)_{J^{(B)}=0}<0$. Interestingly, a longer channel is more effective in maintaining flow of the driven species against its concentration gradient, as $\log _{10}\left(c_{1}^{(B)} / c_{2}^{(B)}\right)_{J^{(B)}=0}$ shifts to the left with increasing channel length. If flow of species $B$ is antiparallel to its concentration gradient its transport-related entropy production is negative $\dot{S}^{(B)}=J^{(B)} \Delta \mu^{(B)}<0$, where $\Delta \mu=\ln \left(c_{1}^{(B)} / c_{2}^{(B)}\right)$ is the difference of the chemical potentials. This negative entropy production is more pronounced in longer channels [16]. It results from the fact that a concentration gradient of a driving species has more options of building up entropic forces in a longer channel, i.e., this entropy-mediated coupling of the two species is stronger in the longer channel, which increases the efficiency of the driving species. Note that this feature of longer channels may also explain why flow of the driven species in Fig. 1 is only moderately dependent on channel length and not, as one would intuitively expect, inversely related.

Up to here scenarios were investigated where there is a driven species profiting from a driving one, however at the cost of the latter. The question arises whether there exist situations where both species cooperate, i.e., both species drive each other mutually. This implies that flow of either species is larger in the presence of a concentration gradient of the other species when compared to a vanishing gradient of the latter. Mathematically this reads

$$
\begin{aligned}
& J^{(B)}\left(\Delta c^{(A)}, \Delta c^{(B)}\right)-\underbrace{J^{(B)}\left(0, \Delta c^{(B)}\right)}_{\text {no gradient of } A}>0, \\
& J^{(A)}\left(\Delta c^{(A)}, \Delta c^{(B)}\right)-\underbrace{J^{(A)}\left(\Delta c^{(A)}, 0\right)}_{\text {no gradient of } B}>0 .
\end{aligned}
$$

Figure 4 shows that there are four zones describing the degree of competition and cooperation of both species. Depending on the sign of the flow differences in Eqs. (7) we have competition at the cost of both (both signs negative), either promotion of one species (positive sign) at the cost of the other (negative sign), or cooperation (both flow differences with positive sign). Note that the sign of the flow difference in Eqs. (7) is the same as that of the corresponding entropy production difference

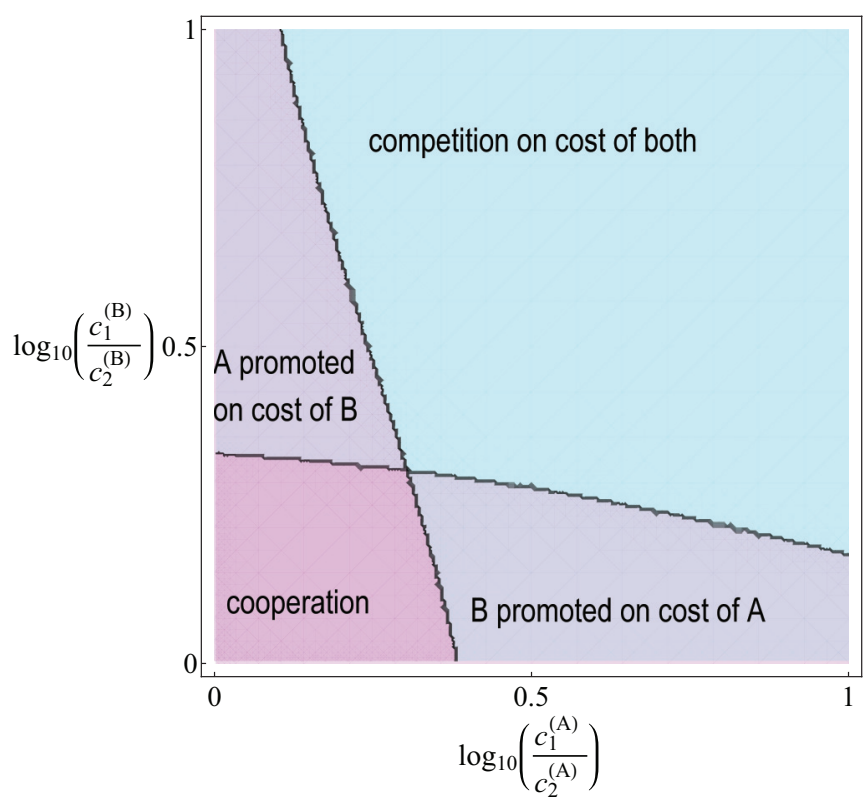

FIG. 4. (Color) Cooperation and competition of two species for parallel directing concentration gradients. Concentration in bath 2 is held constant for both species, $k_{+} c_{2}^{(A)}=k_{+} c_{2}^{(B)}=k_{+} c_{e q}^{(A / B)}=0.1$, whereas that of bath 1 is increased from equilibrium. Channel length is $N=3$, attractive particle channel interactions are chosen as $\Phi_{i}^{(A)} \equiv$ -3 and $\Phi_{i}^{(B)} \equiv-4$, and we set $k_{-}=1$ for both species. Species $B$ is promoted by $A$ when its flow is higher in the presence of a concentration gradient of $A$ compared to the situation in its absence [see Eq. (7)].

$\Delta \dot{S}^{(A, B)}=\Delta J^{(A, B)} \Delta \mu^{(A, B)}$. To understand the phenomena of cooperation one has to consider the different entropic effects driving and hampering the particle of one species, e.g., $B$. One is the concentration gradient of $B$ and the other two effects are related to the gradient of $A$, namely, an entropic force favoring occupation configurations that bias transitions of $B$ in the direction of the gradient of $A$. The other effect is directed oppositely and results from channel blocking by $A$. This force, which is proportional to the gradient of $A$, hampers flow of $B$. For a sufficiently small range of the gradient of $A$, blocking of $B$ is inferior to supporting flow, i.e., $B$ is promoted by $A$. The same holds vice versa for the effect of the gradient of $B$ on flow of $A$. In the overlap of concentration ranges in which both species are mutually promoted by the other, cooperation is present.

It is tempting to try to describe the coupling between the flow of both species in terms of flows and forces in thermodynamic systems that, although being out of equilibrium, retain a notion of local stationarity. Using the chemical potential as the driving force of the particle flux $\Delta \mu \propto \ln \left(c_{1} / c_{2}\right)$, this would lead to

$$
\begin{aligned}
& J^{(A)}\left(\Delta c^{(A)}, \Delta c^{(B)}\right)=L^{(A, A)} \Delta \mu^{(A)}+L^{(A, B)} \Delta \mu^{(B)}, \\
& J^{(B)}\left(\Delta c^{(A)}, \Delta c^{(B)}\right)=L^{(B, A)} \Delta \mu^{(A)}+L^{(B, B)} \Delta \mu^{(B)},
\end{aligned}
$$

with Onsager's theorem stating the equivalence of the cross coefficients

$$
L^{(B, A)}=L^{(A, B)} .
$$




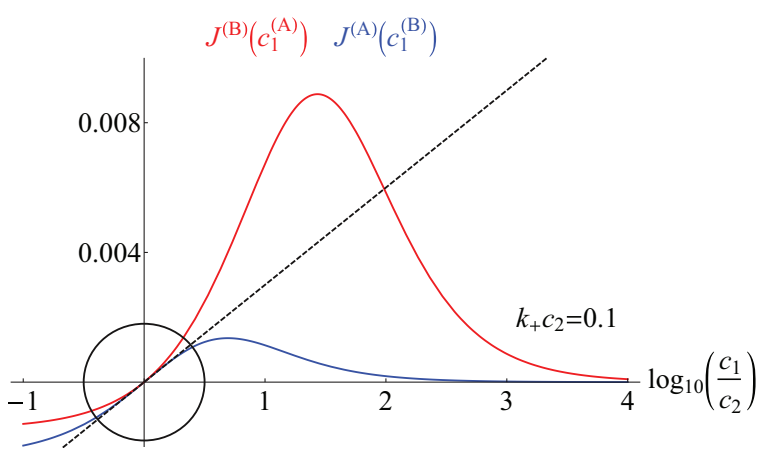

FIG. 5. (Color) Flow of a driven species [ $B$ (red) and $A$ (blue)], with equal concentrations in both baths $\left(k_{+} c_{1}=k_{+} c_{2}=0.1\right)$, vs the gradient of the driving (reciprocal) species. Its concentration in bath 2 is held constant $k_{+} c_{2}=0.1$. We set $k_{-}=1$ for either species and channel length is $N=2$. Species $B$ exhibits an attractive channel interaction $\Phi_{i}^{(B)} \equiv-2$ and species $A$ is inert $\Phi_{i}^{(A)} \equiv 0$. The circle marks the concentration range near the equilibrium point $k_{+} c_{1}$. The dashed line, as it is the tangent of both the red and blue curves at this point, demonstrates that flow of both species exhibits the same linear dependence on the reciprocal concentration gradient (of the driving species), which is the quintessence of the Onsager theorem.

A comparison with the relation (7) shows that in our case there should be, depending on the sign of the cross coefficient, either only cooperation (positive sign) or competition (negative sign), respectively, independent of concentration. However, as shown above, this is not the case.

That Onsager's relation breaks down here (for a more detailed discussion see Ref. [16]) becomes evident not only by the dependence of cooperation and competition on the concentration gradients of the respective species. It is also reflected by the dependence of the driving effectiveness on the particlechannel interaction. To study this more closely, we consider two species with different particle-channel interactions. Reciprocally the flow of one species is analyzed as a function of its partner's concentration gradient (Fig. 5), whereas its own gradient is set to zero. Near equilibrium the reciprocal dependence of flow of one species on the gradient of the other is identical, as Onsager's relation (9) predicts $J^{(B)}\left(\Delta c^{(A)}=x, \Delta c^{(B)}=0\right) \equiv$ $J^{(A)}\left(\Delta c^{(A)}=0, \Delta c^{(B)}=x\right)$. However, this relation only holds in a small range near equilibrium, encircled in Fig. 5, but fails for a further increase of the driving gradient, when a binding site of the driven species increases its transport effectiveness. What remains to be analyzed is how this selectivity of one species is related to cooperation and competition of the two species.

In a Markovian model of nanochannel transport that is numerically exact solvable we could demonstrate how transport of a driving species promotes that of a driven one by building up biasing entropic constrains. However, these entropic constraints may also mutually hamper flow due to crowding, which depends on particle concentrations and particle-channel interaction. As these parameters determine to what extent entropic constraints promote or hamper flow of each species, they also define whether two species mutually cooperate, whether one species promotes transport of the other at its own cost, or whether there is pure competition hampering transport of both species.

The authors' research was supported by the Deutsche Forschungsgemeinschaft (Grant No. SFB688 (TP B05) to W.R.B.) and the Bundesministerium für Bildung und Forschung (Grant No. BMBF01 EO1004 to W.R.B.).
[1] S. M. Iqbal, D. Akin, and R. Bashir, Nat. Nanotechnol. 2, 243 (2007).

[2] T. Jovanovic-Talisman, J. Tetenbaum-Novatt, A. S. McKenney, A. Zilman, R. Peters, M. P. Rout, and B. T. Chait, Nature (London) 457, 1023 (2009).

[3] A. M. Berezhkovskii, M. A. Pustovoit, and S. M. Bezrukov, J. Chem. Phys. 109, 9952 (2002).

[4] A. M. Berezhkovskii, M. A. Pustovoit, and S. M. Bezrukov, J. Chem. Phys. 119, 3943 (2003).

[5] W. R. Bauer and W. Nadler, J. Chem. Phys. 122, 244904 (2005).

[6] A. M. Berezhkovskii, M. A. Pustovoit, and S. M. Bezrukov, Phys. Rev. E 80, 020904 (2009).

[7] L. Dagdug, M.-V. Vazquez, A. M. Berezhkovskii, and S. M. Bezrukov, J. Chem. Phys. 133, 034707 (2010).

[8] A. M. Berezhkovskii and S. M. Bezrukov, Chem. Phys. 319, 342349 (2005).

[9] A. M. Berezhkovskii and S. M. Bezrukov, Biophys. J. 88, L17 (2005).
[10] W. R. Bauer and W. Nadler, Proc. Natl. Acad. Sci. USA 103, 11446 (2006).

[11] A. Zilman and G. Bel, J. Phys.: Condens. Matter 22, 454130 (2010).

[12] W. R. Bauer and W. Nadler, PLoS ONE 5, e15160 (2010).

[13] A. B. Kolomeisky, Phys. Rev. Lett. 98, 048105 (2007).

[14] A. Zilman, S. D. Talia, T. Jovanovic-Talisman, B. T. Chait, M. P. Rout, and M. O. Magnasco, PLoS Comput. Biol. 6, e1000804 (2010).

[15] W. Nadler and K. Schulten, J. Chem. Phys. 84, 4015 (1986).

[16] See Supplemental Material at http://link.aps.org/supplemental/ 10.1103/PhysRevE.88.010703 for determination of the transition matrix, evaluation of entropy production, and the relationship with Onsager's relation.

[17] T. Chou, J. Chem. Phys. 110, 606 (1999).

[18] T. Chou and D. Lohse, Phys. Rev. Lett. 82, 3552 (1999).

[19] P. H. Nelson, J. Chem. Phys. 134, 165102 (2011). 